\title{
Exploring nonlinear pulse propagation, Raman frequency conversion and near octave spanning supercontinuum generation in atmospheric air-filled hollow-core Kagomé fiber
}

\author{
Seyedmohammad Abokhamis Mousavi*, Hans Christian Hansen Mulvad, Natalie Wheeler, Peter \\ Horak, Thomas D. Bradley, Shaif-ul Alam, John Hayes, Seyed Reza Sandoghchi, David Richardson, \\ Francesco Poletti \\ Optoelectronics Research Centre (ORC), University of Southampton, Southampton SO17 1BJ, \\ United Kingdom
}

\begin{abstract}
We have demonstrated Raman frequency conversion and supercontinuum light generation in a hollow core Kagomé fiber filled with air at atmospheric pressure, and developed a numerical model able to explain the results with good accuracy. A solid-state disk laser was used to launch short pulses ( 6ps) at 1030nm into an in-house fabricated hollow core Kagomé fiber with negative core curvature and both ends were open to the atmosphere. The fiber had a $150 \mathrm{THz}$ wide transmission window and a record low loss of $\sim 12 \mathrm{~dB} / \mathrm{km}$ at the pump wavelength. By gradually increasing the pulse energy up to $250 \mu \mathrm{J}$, we observed the onset of different Kerr and Raman based optical nonlinear processes, resulting in a supercontinuum spanning from 850 to $1600 \mathrm{~nm}$ at maximum input power. In order to study the pulse propagation dynamics of the experiment, we used a generalized nonlinear Schrödinger equation (GNLSE). Our simulations showed that the use of a conventional damping oscillator model for the time-dependent response of the rotational Raman component of air was not accurate enough at such high intensities and large pulse widths. Therefore, we adopted a semiquantum Raman model for air, which included the full rotational and vibrational response, and their temperature-induced broadening. With this, our GNLSE results matched well the experimental data, which allowed us to clearly identify the nonlinear phenomena involved in the process. Aside from the technological interest in the high spectral density of the supercontinuum demonstrated, the validated numerical model can provide a valuable optimization tool for gas based nonlinear processes in air-filled fibers.
\end{abstract}

Keywords: Hollow core fiber, nonlinear optics, Raman generation, supercontinuum generation, air-filled hollow core fiber, microstructure fibers.

\section{INTRODUCTION}

During the last decade, demonstration of high peak power pulsed lasers operating in the GW range with high repetition rates in a commercial tabletop form has allowed development of novel applications in, for example, micro-machining ${ }^{1}$, micro-surgery ${ }^{2}$ and biophotonics ${ }^{3}$. However, the ultra-high peak power characteristics of these lasers creates the problem of delivering such pulses in a flexible and reliable way. Conventional solid-core fibers can only manage to deliver pulses with energies in the nanojoule range and come short to handle such high power pulses either due to the low damage threshold in the core material (e.g. typically a silica glass ${ }^{4}$ ) or because the pulse becomes distorted by the nonlinearity of the glass induced by Kerr or Raman processes. A better platform for high power pulse delivery is arguably provided by hollow core fibers, which offer high power delivery thanks to a considerably higher damage threshold and a several orders of magnitude lower nonlinearity ${ }^{5}$. Hollow-Core Photonic Band Gap Fibers (HC-PBGFs) have shown the lowest loss among all hollow core waveguides ${ }^{5}$; however, their high overlap between the optical field and the cladding-glass significantly decreases the damage threshold in these fibers ${ }^{6}$. On the other hand, hollow core fibers with a Kagomé structure and more specifically with a negative-curvature (hypocycloid) core surround have shown a better balance between loss and power handling, with higher damage threshold for ultra-high power delivery than PBG fibers?

Ambient air, that can be found in the core of hollow core fibers, has an over two orders of magnitude lower nonlinear index $\left(\sim 6 \times 10^{-19} \mathrm{~cm}^{2} / \mathrm{W}\right)^{8,9}$ than the glass conventionally used in solid core fibers (i.e. silica) ${ }^{10}$. Despite this, at very high peak powers the nonlinear behavior of air can affect the propagation of pulses and introduce amongst other things,

*sam1e12@soton.ac.uk 
spectral broadening, pulse distortion and ultimately loss of power, all of which are typically detrimental in a power delivery application. For some applications however this can be useful, and nonlinear processes can be exploited for pulse modification, creation of new frequencies and supercontinuum generation (SG) with very high power density due to significant high damage threshold of the gas ${ }^{6}$. The simplicity of using HC-KFs filled with ambient air, combined with the very long light-gas interactions that can be achieved in these fibers has motivated researchers to explore the potential applications of such air-filled fibers. Recently, experimental and theoretical works conducted in this field have demonstrated the capability of air-filled HC-KFs for a variety of nonlinear applications such as: pulse compression ${ }^{11}$, frequency comb generation and supercontinuum generation ${ }^{12}$ to name just a few.

In this work, we explore the nonlinear behavior of an ambient air-filled HC-KF and demonstrate a strong long range frequency conversion due to vibrational Raman scattering of $\mathrm{N}_{2}$, which evolves towards a broadband supercontinuum as the energy of the pulses is increased. Despite the use of pulses with lower peak power as compared to previous works ${ }^{12}$, the use of a record low loss HC-KF ${ }^{13}$ has facilitated the use of a fairly long interaction path $(13.6 \mathrm{~m})$. This, combined with the use of ps rather than fs pulses helped us favorably balancing nonlinear processes. Furthermore, in order to be able to theoretically reproduce the results over such a board spectra range, we have adopted a semi-quantum model to simulate the nonlinear behavior of air with a good precision. Using this model, the nonlinear dynamic of pulse propagation has been simulated in a very good agreement with experimental results and provides a useful tool for analyzing the nonlinear behavior of air.

\section{EXPERIMENTAL SETUP AND RESULTS}

\subsection{Fiber characteristics}

In this experiment, air at atmospheric pressure contained inside a long length ( 14 $\mathrm{m}$ scale) of fiber interacts with a high power laser pulse. As it acts both as light confiner and as gas container, the hollow fiber obviously plays a key role in this experiment and its optical and geometric characteristics are of critical importance in understanding the nonlinear processes occurring to generate the supercontinuum. Although there are different types of hollow core fibers, from a simple capillary with high loss to HC-PBGFs with sophisticated structure but very low loss optical guidance, negativecurvature Kagomé structure has proven to be one of the best options for high power laser beam delivery. The ultra-low overlap between core-field and cladding-glass in HC-KF increases the damage threshold beyond that of other fibers' limitations ${ }^{7}$, which allows exploration of the behavior of gas in interaction with ultra-high power pulses over extremely long interaction lengths. Recently introduced negative-curvature core boundary HC-KFs ${ }^{14,15}$ have dramatically reduced the attenuation of this type of hollow core fiber and further enhanced the high power delivery capability. Furthermore, the wide bandwidth transmission of these fibers makes them one of the best options for broadband nonlinear process such as large Raman shift, frequency comb generation and SG in gases ${ }^{6}$.

Here, we used an in-house-made 7-cell HC-KF with a large bandwidth centered around a wavelength of $1 \mu \mathrm{m}$ This fiber demonstrated a new record low loss and record low bend loss for HC-KFs ${ }^{13}$. Figure 1 shows the scanning electron micrograph (SEM) of the cross section of the fiber, which has a core diameter of $43 \mu \mathrm{m}$ and an average core wall thickness of $\sim 375 \mathrm{~nm}$. The minimum loss of this fiber is $12.3 \mathrm{~dB} / \mathrm{km}$ at $1010 \mathrm{~nm}$ and the $3 \mathrm{~dB}$ bandwidth is $150 \mathrm{~nm}$, while it has a very wide low loss transmission window from $850 \mathrm{~nm}$ to $1700 \mathrm{~nm}$ with average loss of less than 200 $\mathrm{dB} / \mathrm{km}$. This makes this fiber a suitable host for the observation of wideband nonlinear processes (Figure 1). According to our simulations, the percentage of core-field in the cladding-glass for this fiber is of the order of $10^{-5}$, which could theoretically lead to an ultra-high damage threshold (two order of magnitude higher than hollow core PBG fiber) ${ }^{6}$. This fiber has a higher order transmission window below $800 \mathrm{~nm}$ in addition to fundamental one that is presented in Figure 1. Although these characteristics of HC-KF provide an excellent opportunity to explore multi-transmission band nonlinear processes and applications, this work only focused on the nonlinearity observed in the fundamental transmission window.

\subsection{Experimental setup}

Considering the characteristics of this fiber discussed above, which include record low attenuation, low bend loss and large mode field diameter, it is clearly an excellent candidate for high power delivery applications (e.g. industrial machining or surgery). Hence, we have explored the pulse propagation characteristics in an atmospheric air-filled fiber sample at the wavelength of $1 \mu \mathrm{m}$ most commonly used for machining applications. The free space launch setup, used to couple picosecond range laser pulses into the HC-KF, is shown in Figure 2. A commercially available TruMicro $5050^{\odot 16}$ laser generates pulses of $\sim 6 \mathrm{ps}$ duration, $200 \mathrm{kHz}$ repetition rate at $1030 \mathrm{~nm}$ and a maximum energy of $250 \mu \mathrm{J}$ per pulse 
(average power $50 \mathrm{~W}$ ). The output of the TruMicro laser is a collimated beam, and the $\mathrm{M}^{2}$ value is 1.08 . To couple the laser pulses into the fiber, we use a coupling system composed of a variable beam expander (Jenoptik $1 \mathrm{x}-4 \mathrm{x}$ beam expander, AE 1614, 1030-1080 nm, transmission $\geq 97 \%$ ) followed by a focusing lens (Thorlabs LA4148-B-ML, fused silica plan-convex lens with $50 \mathrm{~mm}$ focal length, BBAR coating 650-1050 nm).

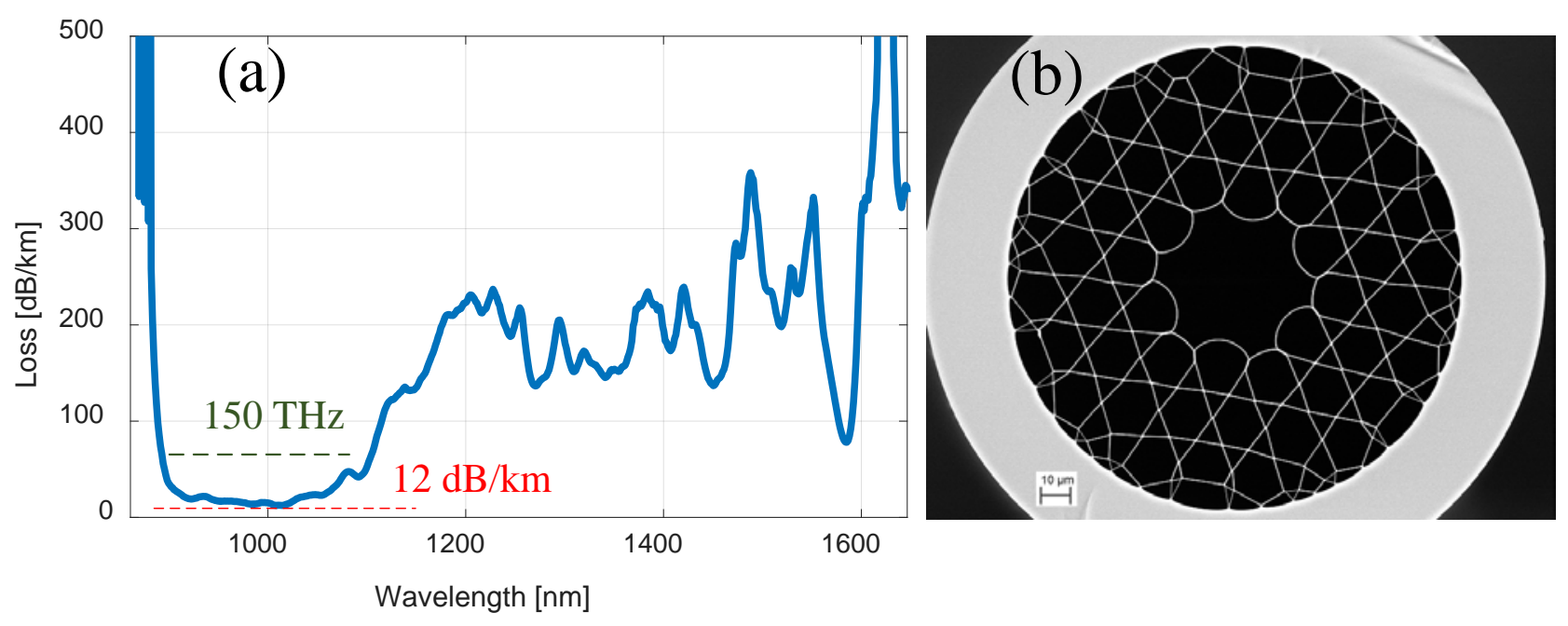

Figure 1. (a) Loss profile of our HC-KF, (b) Scanning electron microscope image of the fiber.

To launch as much light as possible into the fundamental mode of the fiber, the fiber input facet is positioned using a micro-meter positioning stage at the focal point of the focusing lens. The beam expander is used to adjust the width of the collimated beam, such that a beam waist with the optimum width for a fundamental mode launch is obtained at the position of the fiber input. The beam expander is fine-tuned by monitoring and maximizing the output power of the fiber, which indicates an optimum fundamental mode launch due to the higher propagation loss of higher-order modes. As an additional precaution, a camera is used to monitor and detect scattered light from the fiber input facet. The amount of scattered light from the glass microstructure is minimized by fine-tuning the fiber input position, which further reduces the risk of damage to the input facet. The HC-KF is loosely coiled to a $\sim 40 \mathrm{~cm}$ diameter to reduce the induced bend loss. The output power of the fiber is measured using a power meter (Gentec UP55N-300F-H9-D0). To measure the optical spectrum, a small portion of the output beam is reflected off a beam wedge and coupled into an optical spectrum analyzer (OSA - Ando AQ6317B, 600-1750 nm).

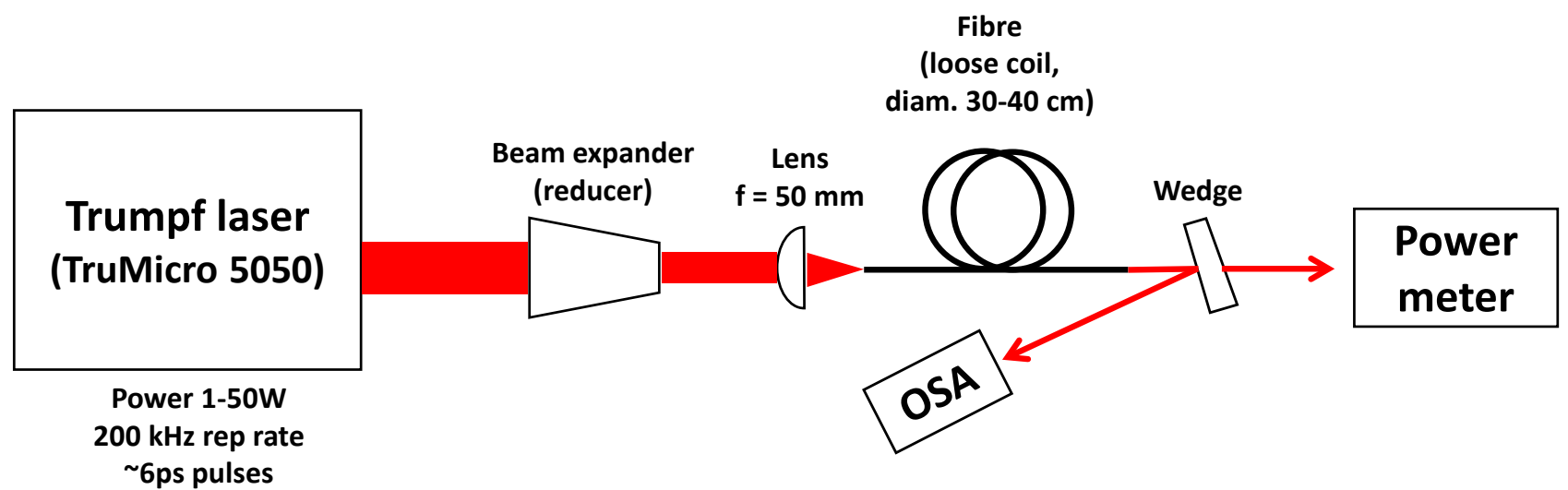

Figure 2. Experimental setup with free space launching mechanism and measurement systems. 


\subsection{Experimental results}

To study the effect of input pulse power on the nonlinear behavior of air and to observe different nonlinear processes, the laser output was coupled into a $13.6 \mathrm{~m}$ length sample of the fiber at a range of powers from fairly low energy pulses up to maximum capacity of the laser, and careful measurements were conducted on the output pulse. The output power spectra are shown in figure 3.

It is clear that the spectral properties of the light at the fiber output depend on input power. At low power pulses (average power $<5 \mathrm{~W}$ ), there is no detectable sign of Raman scattering and the broadening in the pulse occurred through the Kerr effect (self-phase modulation - SPM). As the power increases above $5 \mathrm{~W}$, side bands of the main pump appear, which are in a very good agreement with rotational Raman shifts of Nitrogen and are consistent with the Nitrogen concentration in the air (80\%). As we continue to increase the power, alongside appearance of higher order rotational Stokes and antiStokes shifts next to the pump, the vibrational shift from Nitrogen at $1355 \mathrm{~nm}\left(2330 \mathrm{~cm}^{-1}\right.$ shift $)$ appeared at $>5 \mathrm{~W}$, and even rot-vibrational lines are excited at higher powers $(10 \mathrm{~W})$. In addition, the spectral broadening and spectrum mixing in-between rotational lines are clear, which can be attributed to Kerr effect related phenomena (e.g. SPM, cross phase modulation - XPM, modulation instability - MI). At relatively high powers, the pump and vibrational Raman peaks experience a fast growing broadening effect, which is initiated by the rotational Raman lines of Nitrogen and is accelerated further by the Kerr nonlinearity. These broadening effects continue to extend at higher powers until the spectral side bands of the pump and vibrational Raman lines start overlapping and form a broadband supercontinuum spanning from $850 \mathrm{~nm}$ to $1600 \mathrm{~nm}$. Unlike with single pump broadening schemes, in this form of 'dual pump' SG the whole spectral range is covered by spectral wings of the pump and of its vibrational Raman peaks, which decreases the required pump power for achieving similar bandwidth since the necessary broadening in each 'pump' can be achieved with lower power. In the following section, we analyze the results in more detail by modeling nonlinear behavior of the air in a semi-quantum fashion and simulating the pulse propagation under experimental conditions.

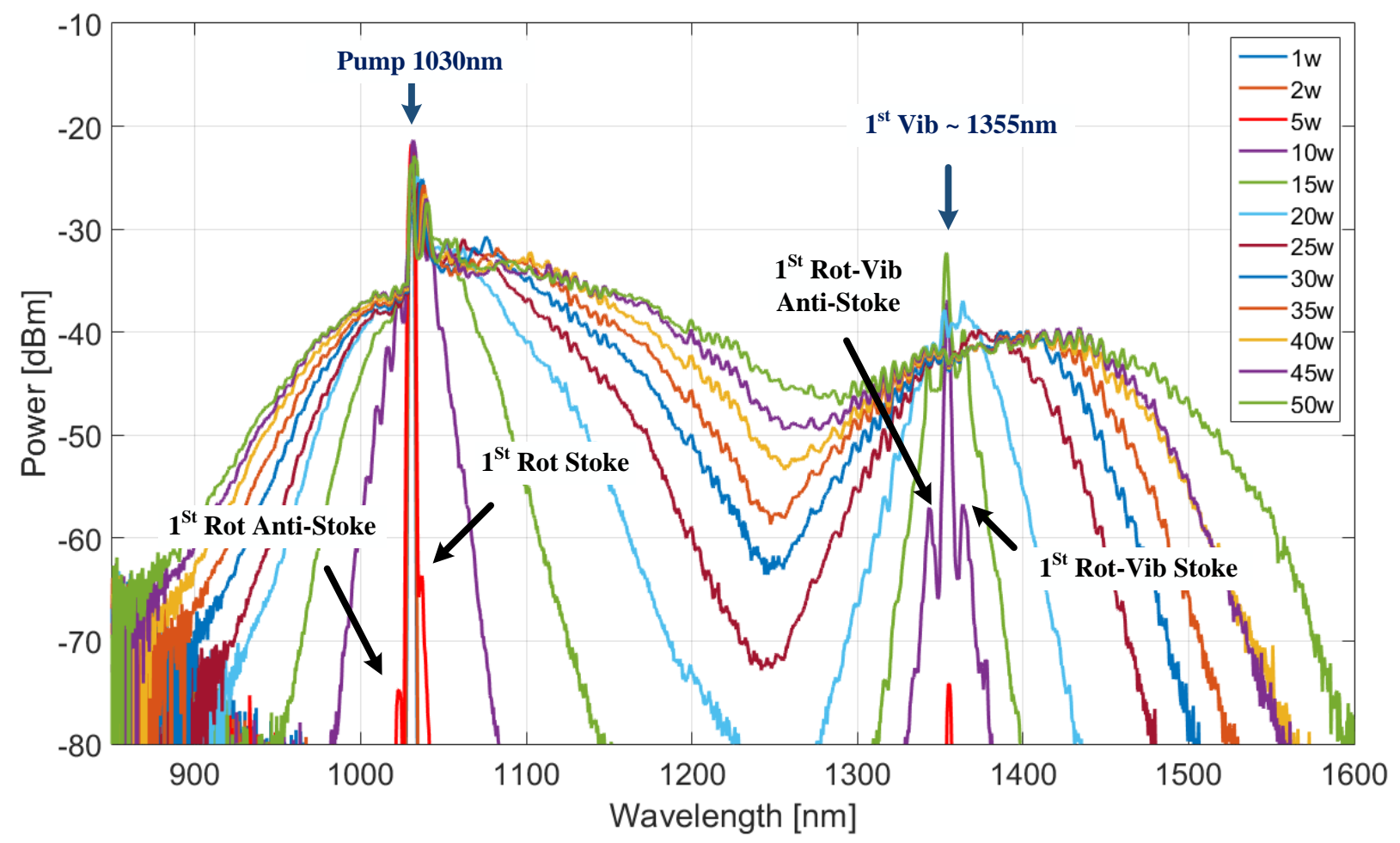

Figure 3. The output power spectrum of a $6 \mathrm{ps}$ pulse with different average powers propagated throughout $13.6 \mathrm{~m}$ of airfilled HC-KF (2nm resolution). 


\section{THEORETICAL MODELING AND DISCUSSION}

\subsection{Theoretical modeling method}

Although modeling the pulse propagation in a complicated gas mixture like air can be challenging, thanks to the domination of Nitrogen ( \%80) and Oxygen ( $\sim 20)$, the complexity of the process can be reduced by modeling only two diatomic molecules. To model the pulse propagation throughout our air-filled HC-KF, the dynamic of the pulse propagation is assumed to be governed by the generalized nonlinear Schrödinger equation (GNLSE) in a frame comoving with the pulse, which includes loss, dispersion and nonlinear properties of the fiber and air as waveguide and core material, respectively ${ }^{17}$ :

$$
\begin{aligned}
& \frac{\partial \tilde{E}(z, \omega)}{\partial z}=-\frac{\alpha}{2} \tilde{E}(z, \omega)+i D(\omega) \tilde{E}(z, \omega)+\cdots \\
& i \sum_{j=1}^{2} K_{j} \gamma_{j}(\omega)\left[1+\frac{\omega-\omega_{0}}{\omega_{0}}\right] F\left\{E(z, t) \int_{-\infty}^{+\infty} R_{j}(T)|E(z, t-T)|^{2} d T\right\}, \quad j=1,2 .
\end{aligned}
$$

where $\tilde{E}(z, \omega)=F\{E(z, t)\}$ denotes the slowly varying envelope of the electromagnetic field in the frequency domain and $j=1,2$ refers to air and glass, respectively. $\alpha(\omega)$ is the loss of the waveguide, which is dominated by the fiber loss profile as shown in figure 1.a and the Rayleigh absorption of air is neglected. $D(\omega)=\beta(\omega)-\beta\left(\omega_{0}\right)-\beta_{1}\left(\omega_{0}\right)\left(\omega-\omega_{0}\right)$, where $\beta(\omega)$ represents the frequency dependent propagation constant of the fundamental mode in the air-filled fiber and $\beta_{n}\left(\omega_{0}\right)$ is the $\mathrm{n}^{\text {th }}$ derivative of the propagation constant at the central frequency of the pulse $\left(\omega_{0}\right)$. As a result, $D(\omega)$ consists of all higher order dispersion values in the frequency domain to fully model the dispersion property of the waveguide. It has been shown that the dispersion of the fundamental mode of a Kagomé fiber can be calculated with a very good approximation by a modified analytical model of a simple capillary due to the similarity of the guidance mechanism and is valid for the spectral range of this experiment ${ }^{18}$ :

$$
\beta(\omega)=\frac{\omega}{c} \sqrt{n_{g a s}^{2}(\omega)-\left(\frac{2.4048 c}{\omega a_{A P}}\right)^{2}} .
$$

here $c$ is the speed of light, $a_{A P}$ is an area-preserving (AP) core diameter of HC-KF and $n_{\text {gas }}(\omega)$ is the refractive index of air which depends on the pressure and temperature of the gas. Figure 4 shows the refractive index of air using the model presented in ${ }^{19}$ and dispersion of air-filled HC-KF using equation 2 at experimental conditions $(T \approx 290 K$, $P=1 \mathrm{~atm})$.

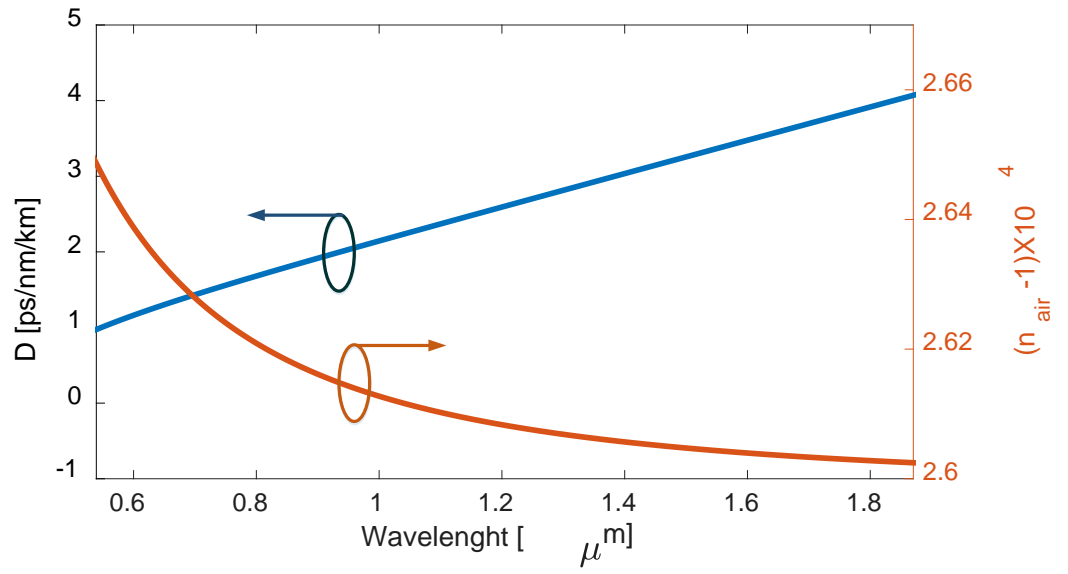

Figure 4. Calculated dispersion profile of the HC-KF in this experiment and air refractive index. 
Furthermore in equation $1, \gamma_{j}(\omega)=\omega_{0} \bar{n}_{j}(\omega) / c A_{\text {eff }}$ with $\bar{n}_{j}(\omega)$ as the nonlinear refractive indices and $A_{\text {eff }}$ as the effective mode area of HC-KF. $R_{j}(t)$ is the nonlinear response of the medium, which can be presented as:

$$
\begin{gathered}
R_{j}(t)=\left(1-f_{R_{j}}\right) \delta(t)+f_{R_{j}} H_{j}(t), \quad j=1,2 . \quad(a) \\
\int_{-\infty}^{+\infty} R_{j}(t) d t=1 . \quad(b)
\end{gathered}
$$

Here, the first part represents the electronic response with instantaneous action on the pulse (Kerr effect), and the second one represents the slower molecular response, $H_{j}(t)$ (Raman response). $f_{R_{j}}$ represents the fractional contribution of Raman into nonlinear response. In order to model the contribution of glass and air in nonlinear response, we have introduced $K_{1}$ and $K_{2}=1-K_{1}$ in equatiuon 1 as the fractional overlap of field with the clad (glass) and core (air), respectively.

A simple classical model for the Raman response $\left(H_{j}(t)\right)$ suggested in the literature ${ }^{9,20}$ is a single damped oscillator (SDO) $H(t) \propto u(t) e^{-t / \tau_{d}} \sin \left(t / \tau_{o}\right)$ where $u(t)$ is the Heaviside function, $\tau_{d}$ is the oscillator damping time, and $\tau_{o}$ is the time constant controlling the oscillation period. Although the simple SDO with $\tau_{d}=32 \mathrm{fs}, \tau_{o}=12.5 \mathrm{fs}$ and $f_{R_{2}}=0.18$ can successfully model a large range of nonlinear effects in silica glass ${ }^{17}$, the attempt for modeling the air-filled fibers regarding its rotational Raman lines by this model can only provide an overall response by taking into account all rotational Raman lines as one broad continuous response, which removes the effect of individual lines in favor of one overlaying envelope. In previous works on air-filled fibers, the air response has been modeled mainly by considering Nitrogen as the dominant gas with $\tau_{d}=77 \mathrm{fs}, \tau_{o}=62.5 \mathrm{fs}$ and $f_{R_{1}}=0.5$ which only takes into the account the rotational

Raman shift of the Nitrogen for their experimental condition ${ }^{9}{ }^{20}$. Such a model can adequately reproduce the first $1 p s$ of the rotational Raman response of Nitrogen (figure 5), which can be enough for some SG by high-power pulses ${ }^{12}$. However, the Raman response of air has a more complex form with extra peaks at longer time scales than the simple model; the combination effect of Kerr with individual Raman lines will be neglected.

\subsection{Semi-quantum model for air}

In our experiments, we have observed a variety of nonlinear processes such as SPM, Stokes and anti-Stokes lines of rotational Raman peaks, vibrational Raman Stokes lines and broadening toward supercontinuum. Although we have tried to simulate and reproduce the experimental results by the SDO model proposed in reference ${ }^{9}$, the complexity and broad range of nonlinear effects cannot be achieved by such a simple model. This discrepancy can be explained by considering the fact that the model does not include the individual rotational lines and their mutual interaction with the Kerr process on broadening of the spectrum. Moreover, this model does not include the vibrational Raman shift, which plays an essential rule in our experiment especially in the SG process. As a result, we use a semi-quantum model for the vibrational Raman response in addition to the full rotational lines of air by considering the contribution of Nitrogen and Oxygen.

In this semi-quantum model, the full rotational Raman response of each gas (Nitrogen or Oxygen) is modeled by summation over individual response function of a manifold of molecular rotational states with rotational quantum number of $\mathrm{J}$, as introduced in ${ }^{8}$. Here, the molecule of gas is considered as a rigid linear rotor, which implies that each rotational state $(J)$ has specific energy as $E=J(J+1) B$ where $B$ is the rotational constant of the rotor. Therefore, by knowing the energy of each state, it is possible to calculate the Raman scattering frequency, since it is equal to the energy difference between initial state and final state of the molecule. Furthermore, the intensity of the spectral lines in rotational Raman response is proportional to the product of the population difference of the coupled states for each transition, the degeneracy of the initial state $(2 \mathrm{~J}+1)$ and the Placzek-Teller coefficient of a rigid rotor. Consequently, the rotational Raman response $H_{R_{k}}(t)$ can be presented as $\left(k=1,2 \text { for } N_{2} \text { and } O_{2}\right)^{8}$ : 


$$
\begin{gathered}
H_{R_{k}}(t)=u(t) C_{R_{k}} e^{\left(-t / \tau_{R_{k}}\right)} \sum_{J} A_{k J} \sin \left(4 \pi B_{k} c(2 J+3)\right), \\
A_{k J}=\left(\rho_{k(J+2)}-\rho_{k J}\right) Z_{k J} \frac{(J+2)(J+1)}{(2 J+3)}, \\
\rho_{k J}=\exp \left[-h c B_{k} J(J+1) / K T\right] . \quad k=1,2 .
\end{gathered}
$$

Here $h, K, T$ are Planck constant, Boltzmann constant and gas temperature, respectively. The rotational constants are $B_{1}=1.99 \mathrm{~cm}^{-1}, B_{2}=1.44 \mathrm{~cm}^{-1}$. The quantum effects due to nuclear statistics (Isotopic effect) are $Z_{1 J}=1$ and 2 for odd and even J, while $Z_{2 J}=1$ and 0 for odd and even J, respectively. The normalization coefficient ( $C_{R_{k}}$ ) defined to normalize the Raman response by equation 3.b. The relaxation factors $\left(\tau_{R_{k}}\right)$ are 61 ps and 68 ps for $N_{2}$ and $O_{2}$, respectively.

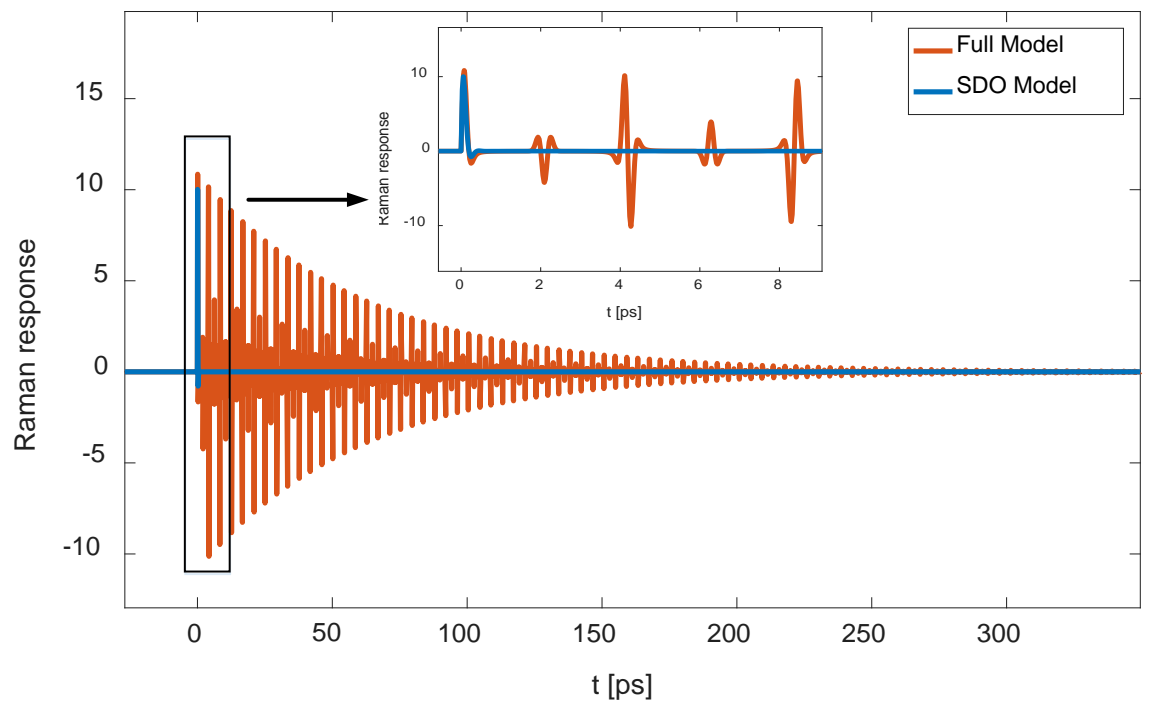

Figure 5. Full model (semi-quantum) and SDO model of rotational Raman response of Nitrogen. The SDO only mimics up to $1 \mathrm{ps}$ of full response.

Following a similar approach and including the rot-vibrational Raman transitions, the vibrational Raman response $H_{R_{k}}(t)$ in Q branch can be calculated $\mathrm{as}^{21}$ :

$$
\begin{gathered}
H_{V_{k}}(t)=u(t) C_{V_{k}} e^{\left(-t / \tau_{V_{k}}\right)} \sum_{J} F_{k J} \sin \left(\omega_{k J} t\right), \\
\omega_{k J} \approx 2 \pi c\left(\bar{\Omega}_{k}-\eta_{k} J(J+1)\right), \\
F_{k J}=Z_{k J}(2 J+1) \exp \left[-h c B_{k} J(J+1) / K T\right], \quad k=1,2 .
\end{gathered}
$$

where $\bar{\Omega}_{k}$ is the central wavenumber of rot-vibrational transition $\left(\bar{\Omega}_{1} \approx 2330 \mathrm{~cm}^{-1}\right.$ and $\left.\bar{\Omega}_{2} \approx 1556 \mathrm{~cm}^{-1}\right)$ and $\eta_{k}$ is the rotational-vibrational interaction constant $\left(\eta_{1} \approx 0.0173 \mathrm{~cm}^{-1}\right.$ and $\left.\eta_{2} \approx 0.0159 \mathrm{~cm}^{-1}\right)$. $C_{V_{k}}$ is the normalization coefficient and $\tau_{V_{k}} \approx \tau_{R_{k}}$. Finally, to achieve a complete Raman response function for air, we combined the rotational and vibrational response of individual gases and considered their concentration in air: 


$$
H_{1}(t)=\sum_{k=1}^{2} \frac{\sigma_{k}}{\bar{n}_{1}}\left[\mu_{R_{k}} H_{R_{k}}(t)+\left(1-\mu_{R_{k}}\right) H_{V_{k}}(t)\right] .
$$

Here, $\bar{n}_{1}$ is the nonlinear refractive index of Nitrogen. $\sigma_{k}$ is the contribution of the individual gas to the Raman response, which can be calculated as the product of molecular concentration ( $80 \%$ for $N_{2}(k=1)$ and $20 \%$ of $\left.\mathrm{O}_{2}(k=2)\right)$ and their nonlinear refractive index. $\mu_{R_{k}}$ is the contribution of rotational part to full response of molecule, since each part of the response function should satisfy the normalization formula (equation 3.b) as much as the full Raman response function, the contribution of vibrational and rotational part should match the experimental data . We found $\mu_{R_{1}}=98.9 \%$ and $\mu_{R_{2}}=100 \%$ to fit our model on experimental data (due to lack of vibrational shift related to Oxygen in our experimental data, we could not calculate its share in our model).

\subsection{Simulation results and discussion}

Using equations 3-6, one can model the Raman response of the air and glass, and by using equations 1,2 one can simulate the pulse propagation, while $f_{R_{1}}$ is needed to be found since the suggested value in ${ }^{9}$ for SDO model is not valid for the new model. By using nonlinear refractive index $(\bar{n})$ of Nitrogen and Oxygen from reference ${ }^{8}$ and silica from reference ${ }^{17}$, respectively, we found $f_{R_{1}}=0.7$ can achieve the best fit to our experimental data and is not far away from the value reported in $^{9}$. Figure 6 shows output spectrum averaged over 20 simulations for individual pulse power alongside the experimental results. Here, the simulation has been performed by the split-step Fourier method with adaptive step size and special care has been taken to eliminate the circulating Raman peaks due to FFT convolution circulating effect from large Raman frequency shifts in simulation window. The simulation results show a very good agreement with experimental data from very low power to fairly high one $\left(\mathrm{P}_{\text {avreg }}<40 \mathrm{~W}\right)$, while at very high powers there are some discrepancies that can be possibly due to guidance distortion and higher loss in glass and gas.

\section{CONCLUSION}

We have used a hollow core Kagomé fiber with negative-curvature core walls and the lowest loss ever reported for this type of fiber to investigate the nonlinear behavior of ambient air in a long light-air interaction path. Using a commercial ps high power laser (TruMicro $5050^{\circ}$ ), we have lunched 6 ps pulses with maximum output energy of $250 \mu \mathrm{J}$ per pulse at $1030 \mathrm{~nm}$ into $13.6 \mathrm{~m}$ of the sample fiber. The output spectrum has shown very close side bands for low average power pulses (5W) in accordance with rotational Raman shift of Nitrogen. At moderate average power pulses (10 W), the $1^{\text {st }}$ Stokes vibrational Raman of Nitrogen introduced a large frequency conversion, while a broadening happened around pump and $1^{\text {st }}$ vibrational peak for higher powers. At maximum power, we could achieve a supercontinuum from 850 to $1600 \mathrm{~nm}$. To model the nonlinear response of air including the vibrational and rotational Raman scattering, we have adopted a semi-quantum model including thermodynamic condition of air. Our model can not only reproduce the air response for long time scale but can also be used in conjunction with the GNLSE for simulating the dynamic of pulse propagation in the air-filled fiber. Using this model, we have simulated the air-filled HC-KF and achieved a good agreement with experimental results. For future work, we intend to investigate the discrepancy between the model and experimental data for even higher peak power pulses and rotational Raman frequency shifts.

\section{ACKNOWLEDGEMENT}

The authors would like to thank Dr Russell S Minns for constructive discussions regarding the air spectroscopy. 

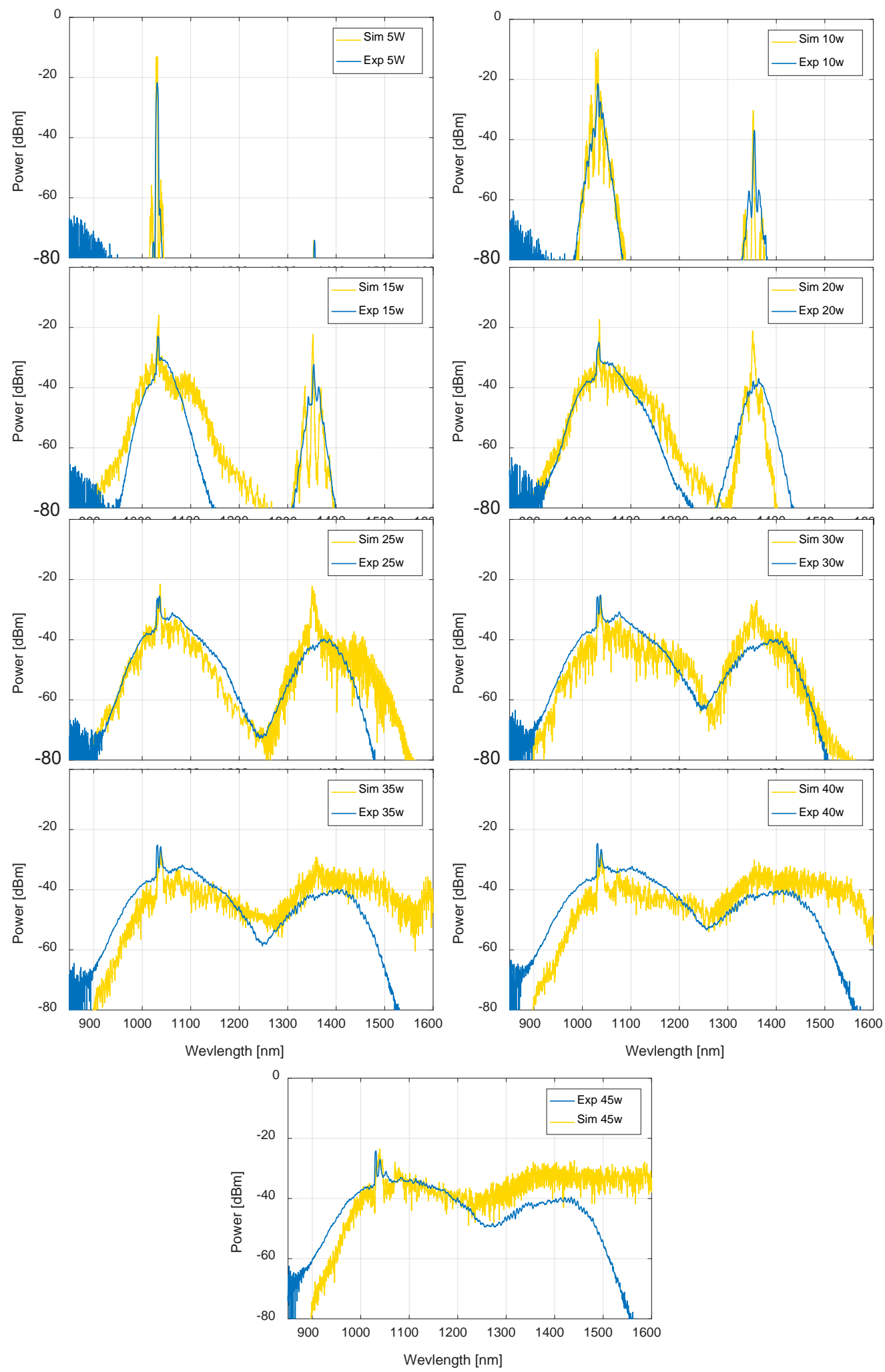

Figure 6. Output spectrum of $6 p s$ pulse passed thru $13.6 \mathrm{~m}$ of atmospheric air-filled HC-KF for different average powers. The experimental results (blue) presented alongside the semi-quantum model simulation (yellow). 


\section{REFERENCES}

[1] Chichkov, B. N., Momma, C., Nolte, S., von Alvensleben, F., and Tünnermann, A., "Femtosecond, picosecond and nanosecond laser ablation of solids," Applied Physics A 63, 109-115 (1996).

[2] Hoy, C. L., Ferhanoğlu, O., Yildirim, M., Piyawattanametha, W., Ra, H., Solgaard, O., and Ben-Yakar, A., "Optical design and imaging performance testing of a 9.6-mm diameter femtosecond laser microsurgery probe," Opt. Express 19, 10536-10552 (2011).

[3] Gu, M., Bird, D., Day, D., Fu, L., and Morrish, D., [Femtosecond Biophotonics: Core Technology and Applications], Cambridge University Press, 2010.

[4] Smith, A. V. and Do, B. T., "Bulk and surface laser damage of silica by picosecond and nanosecond pulses at 1064 nm," Appl. Opt. 47, 4812-4832 (2008).

[5] Poletti, F., Petrovich Marco, N., and Richardson David, J., "Hollow-core photonic bandgap fibers: technology and applications," Nanophotonics 2, 315 (2013).

[6] Russell, P. S. J., Hölzer, P., Chang, W., Abdolvand, A., and Travers, J. C., "Hollow-core photonic crystal fibres for gas-based nonlinear optics," Nat. Photonics 8, 278-286 (2014).

[7] Wang, Y., Alharbi, M., Bradley, Thomas D., Fourcade-Dutin, C., Debord, B., Beaudou, B., Gerôme, F., and Benabid, F., "Hollow-core photonic crystal fibre for high power laser beam delivery," High Power Laser Science and Engineering 1, 17-28 (2013).

[8] Nibbering, E. T. J., Grillon, G., Franco, M. A., Prade, B. S., and Mysyrowicz, A., "Determination of the inertial contribution to the nonlinear refractive index of air, N2, and O2 by use of unfocused high-intensity femtosecond laser pulses," J. Opt. Soc. Am. B 14, 650-660 (1997).

[9] Mlejnek, M., Wright, E. M., and Moloney, J. V., "Dynamic spatial replenishment of femtosecond pulses propagating in air," Opt. Lett. 23, 382-384 (1998).

[10] Kim, K. S., Reed, W. A., Quoi, K. W., and Stolen, R. H., "Measurement of the nonlinear index of silica-core and dispersion-shifted fibers," Opt. Lett. 19, 257-259 (1994).

[11] Debord, B., Alharbi, M., Vincetti, L., Husakou, A., Fourcade-Dutin, C., Hoenninger, C., Mottay, E., Gérôme, F., and Benabid, F., "Multi-meter fiber-delivery and pulse self-compression of milli-Joule femtosecond laser and fiber-aided laser-micromachining," Opt. Express 22, 10735-10746 (2014).

[12] Debord, B., Gérôme, F., Hoenninger, C., Mottay, E., Husakou, A., and Benabid, F., "Milli-Joule energy-level comb and supercontinuum generation in atmospheric air-filled inhibited coupling Kagome fiber," in CLEO: 2015 Postdeadline Paper Digest, (Optical Society of America, 2015), JTh5C.4.

[13] Wheeler, N. V., Bradley, T. D., Hayes, J. R., Gouveia, M. A., Chen, Y., Sandohgchi, S. R., Poletti, F., Petrovich, M. N., and Richardson, D. J., "Low Loss Kagome Fiber in the $1 \mu \mathrm{m}$ Wavelength Region," in Advanced Photonics 2016 (IPR, NOMA, Sensors, Networks, SPPCom, SOF), OSA Technical Digest (online) (Optical Society of America, 2016), SoM3F.2.

[14] Wang, Y. Y., Wheeler, N. V., Couny, F., Roberts, P. J., and Benabid, F., "Low loss broadband transmission in hypocycloid-core Kagome hollow-core photonic crystal fiber," Opt. Lett. 36, 669-671 (2011).

[15] Wang, Y., Couny, F., Roberts, P. J., and Benabid, F., "Low loss broadband transmission in optimized coreshape kagome hollow-core PCF," in Conference on Lasers and Electro-Optics 2010, OSA Technical Digest (CD) (Optical Society of America, 2010), CPDB4.

[16] "TruMicro Series 5000", retrieved http://www.uk.trumpf.com/en/products/laser-technology/products/solidstate-lasers/short-pulsed-lasers/trumicro-series-5000.html.

[17] Agrawal, G. P., [Nonlinear Fiber Optics], Academic Press, 2007.

[18] Finger, M. A., Joly, N. Y., Weiss, T., and Russell, P. S., "Accuracy of the capillary approximation for gas-filled kagome-style photonic crystal fibers," Opt. Lett. 39, 821-824 (2014).

[19] Börzsönyi, A., Heiner, Z., Kalashnikov, M. P., Kovács, A. P., and Osvay, K., "Dispersion measurement of inert gases and gas mixtures at $800 \mathrm{~nm}$," Appl. Opt. 47, 4856-4863 (2008).

[20] Gorbach, A. V. and Skryabin, D. V., "Soliton self-frequency shift, non-solitonic radiation and self-induced transparency in air-core fibers," Opt. Express 16, 4858-4865 (2008).

[21] Zheltikov, A. M., "Raman response function of atmospheric air," Opt. Lett. 32, 2052-2054 (2007). 